\title{
Quantitative measurement of airway dimensions using ultra-high resolution computed tomography
}

\section{$\operatorname{AUTHOR}(S)$ :}

Tanabe, Naoya; Oguma, Tsuyoshi; Sato, Susumu; Kubo, Takeshi; Kozawa, Satoshi; Shima, Hiroshi; Koizumi, Koji; ... Muro, Shigeo; Togashi, Kaori; Hirai, Toyohiro

\section{CITATION:}

Tanabe, Naoya ... [et al]. Quantitative measurement of airway dimensions using ultra-high resolution computed tomography. Respiratory Investigation 2018, 56 (6): 489-496

\section{ISSUE DATE:}

2018-11

\section{URL:}

http://hdl.handle.net/2433/255268

\section{RIGHT:}

(C) 2018. This manuscript version is made available under the CC-BY-NC-ND 4.0 license http://creativecommons.org/licenses/by-nc-nd/4.0/:; This is not the published version. Please cite only the published version.; この論文は出版社版でありません。引用の際には 出版社版をご確認ご利用ください。 


\section{Quantitative measurement of airway dimensions using ultra-high resolution}

2

4 Word count: 2979

5 Author names and affiliations:

6 Naoya Tanabe ${ }^{1}$, Tsuyoshi Oguma ${ }^{1}$, Susumu Sato ${ }^{1}$, Takeshi Kubo ${ }^{2}$, Satoshi Kozawa ${ }^{3}$,

7 Hiroshi Shima $^{1}$, Koji Koizumi ${ }^{3}$, Atsuyasu Sato ${ }^{1}$, Shigeo Muro ${ }^{1}$, Kaori Togashi ${ }^{2}$,

8 Toyohiro Hirai ${ }^{1}$

9

$10{ }^{1}$ Department of Respiratory Medicine, Graduate School of Medicine, Kyoto University,

1154 Kawahara-cho, Shogoin, Sakyo-ku, Kyoto 606-8507, Japan

$12{ }^{2}$ Department of Diagnostic Imaging and Nuclear Medicine, Graduate School of Medicine,

1354 Kawahara-cho, Shogoin, Sakyo-ku, Kyoto 606-8507, Japan

$14{ }^{3}$ Division of Clinical Radiology Service, Kyoto University Hospital, 54 Kawahara-cho,

15 Shogoin, Sakyo-ku, Kyoto 606-8507, Japan

16

$17 \quad$ E-mail Address

18 Naoya Tanabe: ntana@kuhp.kyoto-u.ac.jp

19 Tsuyoshi Oguma: toguma@kuhp.kyoto-u.ac.jp

20 Susumu Sato: ssato@kuhp.kyoto-u.ac.jp

21 Takeshi Kubo: tkubo@kuhp.kyoto-u.ac.jp

22 Satoshi Kozawa: kozawa@kuhp.kyoto-u.ac.jp

23 Hiroshi Shima: hirocima2469@kuhp.kyoto-u.ac.jp

24 Koji Koizumi: koiz@kuhp.kyoto-u.ac.jp 
25 Atsuyasu Sato: atsuyasu@kuhp.kyoto-u.ac.jp

26 Shigeo Muro: smuro@kuhp.kyoto-u.ac.jp

27 Kaori Togashi: ktogashi@kuhp.kyoto-u.ac.jp

28 Toyohiro Hirai: t_hirai@kuhp.kyoto-u.ac.jp

29

30

*Corresponding author: Naoya Tanabe

31 Department of Respiratory Medicine, Graduate School of Medicine, Kyoto University,

3254 Kawahara-cho, Shogoin, Sakyo-ku, Kyoto 606-8507, Japan

33 Tel.: +81 75751 3830; Fa×: +81 757514643

34 E-mail: ntana@kuhp.kyoto-u.ac.jp

35

36 


\section{Abstract}

\section{Background}

Quantitative measurement of airway dimensions using computed tomography (CT) is performed in relatively larger airways due to the limited resolution of CT scans. Nevertheless, the small airway is an important pathological lesion in lung diseases such as chronic obstructive pulmonary disease (COPD) and asthma. Ultra-high resolution scanning may resolve the smaller airway, but its accuracy and limitations are unclear.

\section{Methods}

Phantom tubes were imaged using conventional $(512 \times 512)$ and ultra-high resolution $(1024 \times 1024$ and $2048 \times 2048)$ scans. Reconstructions were performed using the forwardprojected model-based iterative reconstruction solution (FIRST) algorithm in $512 \times 512$ and $1024 \times 1024$ matrix scans and the adaptive iterative dose reduction 3D (AIDR-3D) algorithm for all scans. In seven subjects with COPD, the airway dimensions were measured using the $1024 \times 1024$ and $512 \times 512$ matrix scans.

\section{Results}

52 Compared to the conventional $512 \times 512$ scan, variations in the CT values for air were increased in the ultra-high resolution scans, except in the $1024 \times 1024$ scan reconstructed through FIRST. The measurement error of the lumen area of the tube with 2-mm diameter and 0.5-mm wall thickness (WT) was minimal in the ultra-high resolution scans, but not in the conventional $512 \times 512$ scan. In contrast to the conventional scans, the ultra-high

57 resolution scans resolved the phantom tube with $\geq 0.6-\mathrm{mm}$ WT at an error rate of $<11 \%$. In seven subjects with COPD, the WT showed a lower value with the $1024 \times 1024$ scans versus the $512 \times 512$ scans.

\section{Conclusions}


61 The ultra-high resolution scan may allow more accurate measurement of the bronchioles

62 with smaller dimensions compared with the conventional scan.

$63(248 / 250$ words)

64

65 Keywords

66 Ultra-high resolution computed tomography, Lung, Airway, Chronic obstructive

67 pulmonary disease, Asthma

68

69 Abbreviations

70 AIDR-3D: Adaptive iterative dose reduction 3D

71 CT: Computed tomography

72 COPD: Chronic obstructive pulmonary disease

73 FIRST: forward-projected model-based iterative reconstruction solution

74 HU: Hounsfield unit

75 ROI: regions of interest

76 SD: standard deviation

77 WT: wall thickness 


\section{Introduction}

Chronic obstructive pulmonary disease (COPD) and asthma are major airway diseases

that impose large health problems worldwide [1]. Accurate and sensitive evaluation of the airway structure in relation to its function is important for increasing our understanding of the pathogenetic mechanisms involved and improving management of the disease. Since computed tomography (CT) enables in vivo visualization of the airways less invasively, it is widely used in clinical and research fields [2]. Quantitative structural measurements of the segmental and sub-segmental airways in patients with COPD have allowed determination of the relationships between narrowing of the lumen and impaired airflow limitation [3, 4], as well as between increased wall thickness (WT), respiratory symptoms, and frequent exacerbations [5, 6]. In addition, quantitative CT measurements have shown that increased WT is associated with airflow limitation and responsiveness to inhaled corticosteroid in patients with asthma [7, 8].

In contrast to the large airways, the small airways have been analyzed using histological study, and recently, using microCT [9-12], because the measurement accuracy of the airway dimensions using the conventional CT scan has been validated only in the relatively larger airways [3] with thicker walls [13]. Previous histological studies have shown that the small airways of diameter $<2 \mathrm{~mm}$ are the major site of airflow limitation in patients with COPD [14]. Moreover, in patients with asthma, in addition to the large airways, the small airways are affected by inflammation and mucus plugs [9, 15]. Since obtaining samples for histological study and microCT scanning is too invasive to perform in routine clinical practice, investigators have established a registration method using a pair of inspiratory and expiratory CT scans for assessment of functional small airway disease in vivo [16] and have shown that the functional small airway disease precedes the 
occurrence of emphysema and predicts the decline in pulmonary function in patients with

COPD $[16,17]$. This finding combined with CT data indicating that the luminal area of the $6^{\text {th }}$ generation airways correlated better with airflow limitation than that of the $3^{\text {rd }}$ generation airways in patients with COPD [7], have highlighted the need for accurate and direct measurement of the smaller airway in vivo.

The recent introduction of ultra-high resolution CT has enabled clear visualization of the nodules, airways, vessels, emphysema, and honeycombs in the lungs by increasing spatial resolution up to $0.14 \mathrm{~mm} /$ pixel by using $1024 \times 1024$ and $2048 \times 2048$ matrices without corresponding increase in the radiation dose $[18,19]$. To test the hypothesis that ultrahigh resolution CT scan allows more accurate quantification of the smaller airways than that using conventional CT scan, the present study quantified and compared the dimensions of airway phantom tubes using 1024×1024 and 2048×2048 images obtained using an ultra-high resolution scanner as well as those obtained using conventional 512×512 imaging. Moreover, ultra-high resolution scans were acquired in patients with

117 COPD to test feasibility of the technique under in vivo condition.

2. Patients and methods

\subsection{Ethics Statement}

121 The Ethics Committee of Kyoto University approved the study (approval No. R0311-2, approval date January 14, 2016), and written informed consent was obtained from all participants.

\subsection{Phantom}

The study used two airway phantoms (Kyoto Kagaku Co., Ltd., Kyoto, Japan) including tubes that were made of acrylic resin and surrounded by air. In the first phantom, a tube 
of 2-mm diameter with 0.5-mm WT was analyzed. In the second phantom, six tubes of 3mm diameter with different WT $(0.5,0.6,0.7,0.8,0.9$, and $1.0 \mathrm{~mm})$ were analyzed as reported previously [13]. In addition, variations in the CT values of phantom CT images were estimated by calculating the standard deviation (SD) of CT values in $6 \times 6$-cm square regions of interest (ROI) that contained air in the second phantom. Measurement accuracy of the size of the tubes was validated by using digital calipers.

\subsection{CT Acquisition}

134 CT scans of the phantom were obtained using ultra-high resolution (Aquilion Precision,

135 Cannon Medical, Tokyo, Japan) and conventional scanners (Aquilion One, Cannon 136 Medical, Tokyo, Japan). The phantom was placed perpendicular to the CT slices. In addition to acquisition of conventional images of $512 \times 512$ matrix with $0.5-\mathrm{mm}$ slice thickness, the Aquilion Precision scanner allowed acquisition of images of $1024 \times 1024$ and $2048 \times 2048$ matrices with 0.25 -mm slice thickness through using $0.25 \times 0.25-\mathrm{mm}$ detector elements in the super high-resolution (SHR) mode [18], whereas the Aquilion One scanner provided images of $512 \times 512$ matrix with $0.5-\mathrm{mm}$ slice thickness alone by means of $0.5 \times 0.5-\mathrm{mm}$ detector elements. In both scanners, CT scanning was conducted using the following conditions: $120 \mathrm{kVp}, 240 \mathrm{~mA}$, 0.5-sec exposure time, and 350-mm FOV. For images with matrix sizes of $1024 \times 1024$ and $2048 \times 2048$ in the SHR mode, FOV of $350 \mathrm{~mm}$ allowed 0.34 and $0.17 \mathrm{~mm}$ per pixel resolution in plane. Reconstruction was

146 performed using the enhanced MILD (eMILD), enhanced STANDARD (eSTD), enhanced STRONG (eSTR), and WEAK adaptive iterative dose reduction 3D (AIDR3D), as well as the forward-projected model-based iterative reconstruction solution (FIRST) algorithm [20]. In the process of AIDR-3D, filtered back projection (FBP) data was reconstructed with a high spatial frequency algorithm (FC51) and then mixed with 
the iterative reconstruction process to reduce image noise. The extent of the iterative reconstruction mixture was ranked in increasing order from WEAK, MILD, STD, and STR. Because large mixture in the iterative reconstruction process might diminish the original image texture, the scanners' function to enhance contrast in the images reconstructed by MILD, STD, and STR AIDR-3D, i.e., eMILD, eSTD, and eSTR, respectively, was used. FIRST reconstruction was available for the $512 \times 512$ image obtained using both scanners, and the $1024 \times 1024$ image obtained using the Aquilion Precision scanner. Patients with COPD were scanned using the Aquilion Precision scanner under the same scanning parameters, but auto-exposure control was used to

160 reduce the radiation dose at predetermined level of image noise with standard deviation 161 (SD) of 20 Hounsfield units (HU). Volume CT dose index (CTDI ${ }_{\mathrm{vol}}$ ) was used to estimate the radiation exposure for each scan.

\subsection{Measurements of the dimension of phantom tubes and the airways}

164 The dimensions of tubes and the airways were measured using custom-made software as

165 previously reported [13, 21, 22]. Briefly, the center line of the lumen was determined 3dimensionally, and slice images perpendicular to the center line were reconstructed using trilinear interpolation. On each of the new cross-sectional images from the middle twothirds section of phantom tube and the airways, 128 rays were placed from the center of

169 lumen outward, and the CT values along each ray were calculated. Subsequently, the 170 edges of the airway wall were automatically determined based on the full-width at halfmaximum principle, in which the border between the inside and outside wall was defined as the half difference between the maximum CT value in the wall and the minimum CT value in the lumen. After identification of the airway wall, the luminal area and the WT were calculated and averaged. The measurement error (\%) was calculated using the 
following formula: $100 \times($ CT measurement - actual value $)$ / actual value.

176

177

178

179

180

181

182

183

184

185

186

187

188

\subsection{Pulmonary function test}

In the patients with COPD, spirometry was performed after inhalation of a bronchodilator through Chestac-65V (Chest MI Corp., Tokyo, Japan).

\subsection{Statistics}

The data were expressed as the mean \pm SD. Statistical analysis was performed using $\mathrm{R}$ software [23]. A p-value less than 0.05 was considered to be statistically significant.

\section{Results}

\subsection{Phantom study}

Figure 1A shows a phantom image that included ROI for air. The mean CT values for the ROI of each scan were within $-1000 \pm 1 \mathrm{HU}$, without significant differences of that among scans acquired under different imaging conditions. In contrast, the SD of CT values for air (Figure 1B) was increased with increased matrix size of the image from $512 \times 512$ to 2048×2048, except for the FIRST reconstruction. Among the ultra-high resolution images (1024×1024 and $2048 \times 2048)$, the noise was lowest in the FIRST reconstruction followed by that in the eSTR and WEAK AIDR-3D.

Figure 2A shows that compared with the conventional scanner $(512 \times 512$ matrix, 0.5-mm slice thickness, WEAK AIDR-3D), the phantom tube (diameter, 2 mm; WT, 0.5 mm) was imaged more clearly by using the ultra-high resolution scanner (matrix, 2048×2048; slice thickness, 0.25 mm; reconstruction, eMILD or WEAK AIDR-3D; and matrix, 1024×1024; slice thickness, $0.25 \mathrm{~mm}$; reconstruction, FIRST). The CTDI $\mathrm{vol}_{\mathrm{vl}}$ in the phantom scans using the conventional and ultra-high resolution scanners were 13.7 and 10.4 mGy, respectively. Table 1 shows the measurement errors of the lumen area and WT 
199 of the phantom tube. The measurement error of the lumen area and WT were smaller with

200 the ultra-high resolution scans versus the conventional $512 \times 512$ scans.

In addition, Figure 2 shows differences in the CT value curve along an outward ray from the luminal center through the wall between images of matrix size, $512 \times 512$ and 1024×1024 (2B) and that of reconstructions, eMILD and FIRST (2C). Figure 2B shows that a larger region was identified as the wall in the image with $512 \times 512$ matrix than that in the image with $1024 \times 1024$ matrix. Figure 2C shows that the CT value in the parenchyma adjacent to the wall was lower, and the maximum CT value in the wall was higher in scans reconstructed with eMILD AIDR-3 than those with FIRST; whereas, a smaller region was identified as the wall in scans reconstructed through eMILD than that in those through FIRST.

Figure 3 shows that the measurement errors of WT were increased with decrease

211 in the thickness of the tube walls from 1.0 to $0.5 \mathrm{~mm}$. The measurement errors of WT in

212 the $1024 \times 1024$ FIRST-reconstructed, $1024 \times 1024$ WEAK-reconstructed, and the

$2132048 \times 2048$ WEAK-reconstructed images showed lower proportion $(<11 \%)$ of tubes with

2140.6 to $1.0-\mathrm{mm}$ WT. In the $1024 \times 1024$ and $2048 \times 2048$ images reconstructed through eMILD, eSTD, and eSTR AIDR-3D, the measurement error of WT was small in the tube with $0.5-\mathrm{mm} \mathrm{WT}$, and had negative value in the tubes with $0.8,0.7$, and $0.6-\mathrm{mm} \mathrm{WT}$.

\section{$217 \quad 3.2$ Measurements of dimensions of the airway in humans}

218 Figure 4A shows an example of $1024 \times 1024$ CT scan obtained using the ultra-high 219 resolution scanner (slice thickness, $0.25 \mathrm{~mm}$; reconstruction, FIRST) in a subject with COPD. The white square indicates the $5^{\text {th }}$ generation of the right apical bronchus $\left(\mathrm{B}^{1}\right)$ in the $1024 \times 1024$ image, which was compared with the lower-resolution image of $512 \times 512$ matrix, 0.5-mm slice thickness, and FIRST reconstruction shown in Figure 4B. 
Demographic characteristics of the seven subjects with COPD who underwent evaluation of the airway dimension are described in Table 2. The CTDI ${ }_{\mathrm{vol}}$ measured at the scans was 11.4 $\pm 1.7 \mathrm{mGy}$. The luminal area and WT of the $3^{\text {rd }}, 4^{\text {th }}$, and $5^{\text {th }}$ generations of the right B1 airways were quantified using the $1024 \times 1024$ FIRST-reconstructed CT images in all seven subjects with COPD. In contrast, in the 512×512 FIRST-reconstructed images from two of the seven subjects, the wall of the $5^{\text {th }}$-generation airways could not be segmented automatically. Among the airways quantified using both the $1024 \times 1024$ and $512 \times 512$ matrix images, the WT was substantially lower in the $1024 \times 1024$ images than that in the $512 \times 512$ images (by generation: $3^{\text {rd }}, 1.29$ vs. $1.53 \mathrm{~mm} ; 4^{\text {th }}, 0.86$ vs. $1.23 \mathrm{~mm}$; and $5^{\text {th }}$, 0.76 vs. $1.06 \mathrm{~mm}$, respectively). Bland-Altman plot (Figure 4C) and scatter plot (Figure 4D) showed overestimation of the WT in the $512 \times 512$ scans. Correlations between the WT and pulmonary function for the $3^{\text {rd }}, 4^{\text {th }}$, and $5^{\text {th }}$ generation airways are shown in the Supplementary Figure.

\section{Discussion}

The present study showed that the ultra-high resolution scanner enables more accurate measurements of the lumen area and WT of the phantom tubes and human airways compared with the conventional scanner. The new imaging technique showed potential as a tool for quantitative analyses of the smaller airways than those currently analyzable, with acceptable error rate.

The need for accurate measurement of the small airways in vivo is increasing, as studies using histology and microCT have identified the small airways as the main pathological lesion in patients with COPD and asthma [9, 11, 14, 15, 24]. In addition,

246 previous quantitative measurement of the dimensions of the airway with the 
conventional $512 \times 512$ CT scans showed that the luminal narrowing at the $6^{\text {th }}$ generation of the airways correlated with airflow limitation in patients with COPD better than the narrowing at the $3^{\text {rd }}$ generation airways [3]. However, which generation of the airways has the largest impact on correlation between the lumen area and the pulmonary function remains unclear. The present finding indicating accurate measurement of the lumen area of phantom tube of 2-mm diameter and 0.5-mm WT using the ultra-high resolution versus conventional scan will facilitate development of suitable analytical tool to clarify the issue. This technique enables determination of structure-function relationship in airway disease, locations for site-specific bronchodilators, patients’ response to inhaler therapy, and prognosis in management of patients with COPD and asthma.

The finding of greater measurement error mainly in low WT among measured dimensions of phantom tube confirms the previous finding by Oguma et al [13]. In addition, in ultra-high resolution images, least measurement error in WT was observed in the images with WEAK AIDR-3D and FIRST reconstruction from 1.0 to $0.6 \mathrm{~mm}$ of the actual WT, in order; in contrast, the trend of measurement errors in the eMILD, eSTD, and eSTR-reconstructed images was not consistent, with value of almost $0 \%$ in the images of phantom tube with 1.0 and $0.5-\mathrm{mm}$ WT, whereas, negative values in the images of tube with $0.8,0.7$, and $0.6-\mathrm{mm}$ WT, indicating that the eMILD, eSTD, and eSTR AIDR-3D reconstructions led to underestimated value of the WT. FIRST is one of the full-iterative model reconstruction methods that has been recently proposed for use to improve the signal-to-noise ratio, especially in low-dose CT scans [20]. Currently, although FIRST reconstruction cannot be applied to reconstruct $2048 \times 2048$ matrix images due to the large burden of computation resource, the 
1024×1024 images reconstructed through FIRST showed potential for use in quantitative measurements of the dimensions of the airway for the following reasons. First, the image noise was smaller than that with the other reconstruction algorithms (Figure 1). Second, in the phantom study, the measurement error of the lumen area and WT was substantially improved in the $1024 \times 1024$ matrix image compared to those of the conventional $512 \times 512$ matrix images; however, the improvement in measurement error in increasing order of the images of $1024 \times 1024$ to $2048 \times 2048$ matrix, was very small, possibly because both the $1024 \times 1024$ and $2048 \times 2048$ scans were based on the same $0.25 \times 0.25$-mm size of detector elements, while the conventional $512 \times 512$ scans were based on the larger $0.5 \times 0.5-\mathrm{mm}$ size of detector elements. Third, because the dimension of the airway was evaluated 3-dimensionally and the slice thickness of both the $1024 \times 1024$ and $2048 \times 2048$ scans was $0.25 \mathrm{~mm}$, it was difficult to substantially improve the measurement error by changing the in-plane resolution from $0.34 \mathrm{~mm} /$ pixel to $0.17 \mathrm{~mm} /$ pixel alone.

In patients with COPD, the results revealed that the measured value of WT in the $3^{\text {rd }}, 4^{\text {th }}$, and $5^{\text {th }}$ generation airways using $512 \times 512$ scans (by generation: $3^{\text {rd }}, 1.53$ $\mathrm{mm} ; 4^{\text {th }}, 1.23 \mathrm{~mm} ; 5^{\text {th }}, 1.06 \mathrm{~mm}$ ) were 18,43 , and $40 \%$ higher than those in the $1024 \times 1024$ scans (by generation: $3^{\text {rd }}, 1.29 \mathrm{~mm} ; 4^{\text {th }}, 0.86 \mathrm{~mm} ; 5^{\text {th. }}: 0.76 \mathrm{~mm}$ ). This result is consistent with those in the phantom study, showing a 15 to $40 \%$ measurement error of WT in tubes with 1.0 to $0.7-\mathrm{mm}$ WT using $512 \times 512$ FIRST-reconstructed images (Figure 3). Collectively, these findings and the observed difference in the curve of the

291 CT values in the $512 \times 512$ and $1024 \times 1024$ matrix images (Figure 2B) support previous reports indicating overestimation of the WT with underestimation of the lumen area in quantitative CT measurements of the smaller bronchioles relative to the resolution [2,

294 25]. Studies investigating more accurate measurement of the dimensions of the airway 
using ultra-high resolution imaging are needed to confirm the relationship between the airways’ morphometry, function, and clinical parameters. tube was not higher than that in the conventional scan. Moreover, the CTDIvol in the ultra-high resolution scans of human subjects was $11.4 \pm 1.7 \mathrm{mGy}$, which is equivalent to the reported CTDIvol (12 mGy) in conventional chest CT scans in a large study population [26]. These findings suggested that the radiation exposure in patients undergoing the ultra-high resolution chest scanning is acceptable in the research and clinical setting. measured in the present phantom at the smallest tube diameter of $2 \mathrm{~mm}$ and least WT of $0.5 \mathrm{~mm}$, and the limitation for measurable lumen size could not be determined. Second, the phantom is artificial and does not always reflect the airway in vivo. Ideally, the airway measurements obtained using CT should be validated based on results of histological study. Third, the present study analyzed the airways in patients with COPD, but not in patients with asthma or healthy controls. We could not compare the dimensions of the airway between the COPD, asthma, and healthy control groups. Previous studies using conventional $512 \times 512$ scans showed that remodeling of the

313 airway wall was generally greater in patients with asthma than that in patients with

314 COPD [27]; hence, future study comparing the airway structure between patients with

315 COPD, combined asthma and COPD, and asthma, using ultra-high resolution scanning is required. Finally, in this study, the FIRST reconstruction required longer time (27 min) to generate $1024 \times 1024$ images of the entire lung than the AIDR-3D reconstruction. However, because the new technology targets patients with COPD and asthma with 
stable condition, the image reconstruction time of approximately 30 min can be considered as acceptable for routine clinical practice.

\section{Conclusion}

323 The ultra-high resolution scanner showed superior performance in terms of improving the measurement accuracy of the dimension of phantom tubes. Quantitative analysis of the dimensions of the airway using the new scanner can increase our understanding of the structure-function relationship in patients with lung diseases such as asthma and COPD.

Acknowledgements

This work was supported by a grant from FUJIFILM Medical for collection and analysis of data.

\section{Conflict of interest}

The authors have no conflicts of interest.

\section{References}

337 [1] Adeloye D, Chua S, Lee C, et al. Global and regional estimates of COPD prevalence: Systematic review and meta-analysis. J Glob Health 2015;5:020415.

[2] Pare PD, Nagano T, Coxson HO Airway imaging in disease: gimmick or useful tool? J Appl Physiol (1985) 2012;113:636-46.

341 [3] Hasegawa M, Nasuhara Y, Onodera Y, et al. Airflow limitation and airway dimensions in chronic obstructive pulmonary disease. Am J Respir Crit Care Med 
2006;173:1309-15.

344 [4] Nakano Y, Muro S, Sakai H, et al. Computed tomographic measurements of airway dimensions and emphysema in smokers. Correlation with lung function. Am J Respir Crit Care Med 2000;162:1102-8.

[5] Grydeland TB, Dirksen A, Coxson HO, et al. Quantitative computed tomography: emphysema and airway wall thickness by sex, age and smoking. Eur Respir J 2009;34:858-65.

[6] Han MK, Kazerooni EA, Lynch DA, et al. Chronic obstructive pulmonary disease exacerbations in the COPDGene study: associated radiologic phenotypes. Radiology 2011;261:274-82.

[7] Niimi A, Matsumoto H, Amitani R, et al. Airway wall thickness in asthma assessed by computed tomography. Relation to clinical indices. Am J Respir Crit Care Med 2000;162:1518-23.

[9] Hamid Q, Song Y, Kotsimbos TC, et al. Inflammation of small airways in asthma. J Allergy Clin Immunol 1997;100:44-51.

[10] Tanabe N, Vasilescu DM, McDonough JE, et al. Micro-Computed Tomography

[11] Hogg JC, Chu F, Utokaparch S, et al. The nature of small-airway obstruction in chronic obstructive pulmonary disease. N Engl J Med 2004;350:2645-53. 
emphysema in chronic obstructive pulmonary disease. $\mathrm{N}$ Engl $\mathrm{J}$ Med 2011;365:1567-75.

369

[13] Oguma T, Hirai T, Niimi A, et al. Limitations of airway dimension measurement on images obtained using multi-detector row computed tomography. PLoS One 2013;8:e76381.

[14] Hogg JC, Macklem PT, Thurlbeck WM Site and nature of airway obstruction in chronic obstructive lung disease. N Engl J Med 1968;278:1355-60.

[15] Kuyper LM, Pare PD, Hogg JC, et al. Characterization of airway plugging in fatal asthma. Am J Med 2003;115:6-11.

[16] Galban CJ, Han MK, Boes JL, et al. Computed tomography-based biomarker provides unique signature for diagnosis of COPD phenotypes and disease progression. Nat Med 2012;18:1711-5.

[17] Bhatt SP, Soler X, Wang X, et al. Association between Functional Small Airway Disease and FEV1 Decline in Chronic Obstructive Pulmonary Disease. Am J Respir Crit Care Med 2016;194:178-84.

[18] Hata A, Yanagawa M, Honda O, et al. Effect of Matrix Size on the Image Quality of Ultra-high-resolution CT of the Lung: Comparison of 512 x 512, 1024 x 1024, and 2048 x 2048. Acad Radiol 2018.

[19] Kakinuma R, Moriyama N, Muramatsu Y, et al. Ultra-High-Resolution Computed Tomography of the Lung: Image Quality of a Prototype Scanner. PLoS One 2015;10:e0137165.

[20] Nishiyama Y, Tada K, Nishiyama Y, et al. Effect of the forward-projected modelbased iterative reconstruction solution algorithm on image quality and radiation dose in pediatric cardiac computed tomography. Pediatr Radiol 2016;46:1663-70. 
391

392

393

394

395

396

397

398

399

400

401

402

403

404

405

406

407

408

409

410

[21] Tanabe N, Muro S, Oguma T, et al. Computed tomography assessment of pharmacological lung volume reduction induced by bronchodilators in COPD. COPD 2012;9:401-8.

[22] Oguma T, Hirai T, Fukui M, et al. Longitudinal shape irregularity of airway lumen assessed by CT in patients with bronchial asthma and COPD. Thorax 2015;70:719-24.

[23] R Core Team, R: A Language and Environment for Statistical Computing. URL http://www.R-project.org/. 2015.

[24] Tanabe N, Vasilescu DM, Kirby M, et al. Analysis of airway pathology in COPD using a combination of computed tomography, micro-computed tomography and histology. Eur Respir J 2018;51.

[25] Nakano Y, Wong JC, de Jong PA, et al. The prediction of small airway dimensions using computed tomography. Am J Respir Crit Care Med 2005;171:142-6.

[26] Smith-Bindman R, Moghadassi M, Wilson N, et al. Radiation Doses in Consecutive CT Examinations from Five University of California Medical Centers. Radiology 2015;277:134-41.

[27] Shimizu K, Hasegawa M, Makita H, et al. Comparison of airway remodelling assessed by computed tomography in asthma and COPD. Respir Med 2011;105:1275-83. 


\section{$411 \quad$ Figure captions}

\section{Figure 1. Standard deviation of computed tomography values on phantom images.}

413 (A) Standard deviation (SD) of CT values were measured in regions of interest (yellow

414 square, $6 \times 6 \mathrm{~cm}$ square) that contained air. (B) The SD values were compared between 415 images acquired through the conventional scanner (Aquilion One, 512×512 matrix 416 [AO512]) and the ultra-high resolution scanner (Aquilion Precision, 512×512, 417 1024×1024, and 2048 $\times 2048$ matrices [AP512, AP1024, AP2048]) with five 418 reconstruction algorithms (eMILD AIDR-3D, eSTD AIDR-3D, eSTR AIDR-3D, WEAK 419 AIDR-3D, and FIRST).

Figure 2. Influence of different imaging conditions on the measurement of the dimensions of phantom tubes.

(A) Comparison of images of phantom tube of 2-mm diameter and 0.5-mm wall thickness between the conventional scanner (Aquilion One, 512×512 matrix, 0.5-mm slice thickness, WEAK AIDR-3D reconstruction) and the ultra-high resolution scanner (Aquilion Precision, 2048×2048 matrix, 0.25-mm slice thickness, eMILD and WEAK AIDR-3D reconstructions; 1024×1024 matrix, 0.25-mm slice thickness, and FIRST reconstruction). (B) Comparison of measurement of the wall thickness (WT) between images with $512 \times 512$ and $1024 \times 1024$ matrix size. The actual size of phantom tube

430 includes diameter of $3 \mathrm{~mm}$ and wall thickness of $0.7 \mathrm{~mm}$. CT values along an outward 431 ray from the lumen center through the wall (yellow line) were plotted for each of the 512×512 (red) and 1024×1024 scans reconstructed with FIRST. (C) Comparison of CT value curve along the outward ray (yellow line) between the eMild, AIDR-3D (green), and FIRST (blue) reconstruction methods. The size of both images is $1024 \times 1024$. The 
inner and outer boundaries of the wall were determined based on the full-width at halfmaximum method.

Figure 3. Measurement errors of the wall thickness in phantom tubes of various sizes.

Comparison of the measurement errors of the wall thickness in six phantom tubes between images acquired using the conventional scanner (Aquilion One, 512×512 matrix [AO512]) and the ultra-high resolution scanner (Aquilion Precision, 512×512, 1024×1024, and 2048×2048 matrices [AP512, AP1024, and AP2048]) with five reconstruction algorithms (eMILD, eSTD, eSTR, WEAK AIDR-3D, and FIRST). In all phantom tubes, the internal diameter is $3 \mathrm{~mm}$, and individual wall thickness is 1.0, 0.9, 0.8, 0.7, 0.6, and $0.5 \mathrm{~mm}$.

Figure 4. Measurement of the dimensions of the airway using ultra-high resolution CT in subjects with COPD.

(A) An example of a chest CT scan obtained using the ultra-high resolution scanner (Aquilion Precision, 1024×1024, 0.25-mm slice thickness, and FIRST reconstruction) in a subject with COPD. The white square indicates the $5^{\text {th }}$ generation of the right B1 airway. (B) An example of the $512 \times 512$ matrix scan of the same airway as shown in (A) reconstructed with FIRST. The wall in (B) could not be segmented at the full-width halfmaximum principle method. (C) Bland-Altman plots of the measured luminal area (Ai) and wall thickness (WT) in the $512 \times 512$ and $1024 \times 1024$ matrix scans $\left(\mathrm{Ai}_{512}, \mathrm{WT}_{512}\right.$, $\mathrm{Ai}_{1024}$, and $\mathrm{WT}_{1024}$, respectively). (D) Scatter plot showing the relationship between $\mathrm{WT}_{512}$ and $\mathrm{WT}_{1024}$ (rho $=0.86$ and $\mathrm{p}<0.0001$ by Spearman correlation test). Red, blue, and green colors indicate the $3^{\text {rd }}, 4^{\text {th }}$, and $5^{\text {th }}$ generations of the right $\mathrm{B} 1$ airways. 
Tables

Table 1. Measurement errors of the lumen area and wall thickness in phantom tube

of 2-mm diameter and 0.5-mm wall thickness.

\begin{tabular}{|c|c|c|c|c|}
\hline & AO512 & AP512 & AP1024 & AP2048 \\
\hline \multicolumn{5}{|c|}{ Lumen area } \\
\hline eMILD & -45 & -8 & 8.5 & 12 \\
\hline eSTD & -46 & -8 & 9.8 & 12 \\
\hline eSTR & -34 & -21 & 1 & 1 \\
\hline WEAK & -47 & -27 & -1 & 2 \\
\hline FIRST & -33 & -19 & 0 & NA \\
\hline \multicolumn{5}{|c|}{ Wall thickness } \\
\hline eMILD & 105 & 57 & 11 & -1 \\
\hline eSTD & 118 & 55 & 9 & -4 \\
\hline eSTR & 9 & 53 & 5 & -5 \\
\hline WEAK & 111 & 64 & 28 & 15 \\
\hline FIRST & I & 62 & 27 & NA \\
\hline
\end{tabular}

Measurement error is calculated as $100 \times$ (measured value - actual value) / actual value.

464 The unit for all values is \%. "The calculated value of the wall thickness is unavailable in

465 the $512 \times 512$ scans reconstructed with FIRST because the actual wall thickness is close to 466 the pixel dimension. NA indicates that the FIRST reconstruction method is unavailable.

467 AO512 indicates images of $512 \times 512$ matrix obtained with the Aquilion One scanner.

468 AP512, AP1024, and AP2048 indicate 512×512, 1024×1024, and 2048×2048 matrix 469 images obtained with the Aquilion Precision scanner. 
470 Table 2. Demographic characteristics of seven subjects with chronic obstructive 471 pulmonary disease.

\begin{tabular}{lc}
\hline Age & $76 \pm 6$ \\
Sex ratio M/F & $7 / 0$ \\
Height (cm) & $166 \pm 4$ \\
Weight (kg) & $63 \pm 8$ \\
Smoking history (Former /Current) & $7 / 0$ \\
Pack Years & $55 \pm 26$ \\
FEV 1 (L) & $1.76 \pm 0.41$ \\
FEV (\%pred) & $66 \pm 14$ \\
FVC (L) & $3.46 \pm 0.53$ \\
FEV 1 FVC (\%) & $51 \pm 7$ \\
LAMA use (\%) & $57 \%$ \\
LABA use & $57 \%$ \\
ICS use & $57 \%$
\end{tabular}

472 Data are expressed as the mean $\pm \mathrm{SD} . \mathrm{FEV}_{1}$, Forced expiratory volume in $1 \mathrm{sec}$,

473 FVC, Forced vital capacity; LAMA, Long-acting muscarinic antagonist; LABA, Long474 acting beta agonist; ICS, Inhaled corticosteroid. 
Fig. 1

A

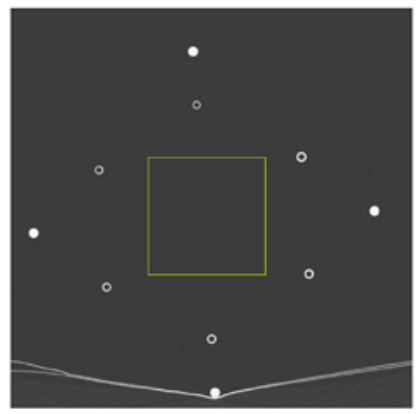

B

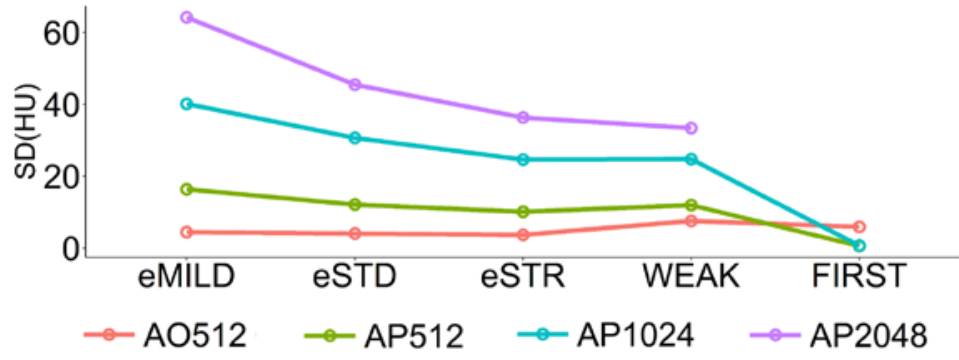

Fig. 2

A

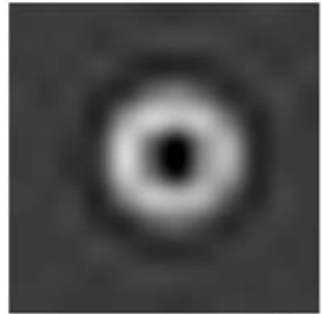

Conventional

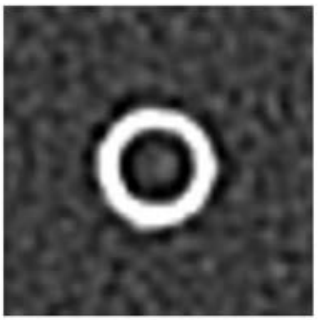

eMILD

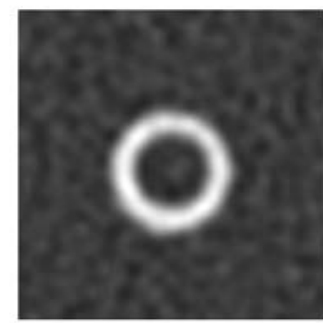

WEAK

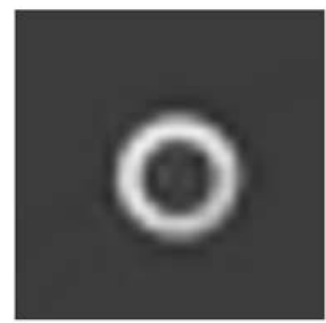

FIRST
B
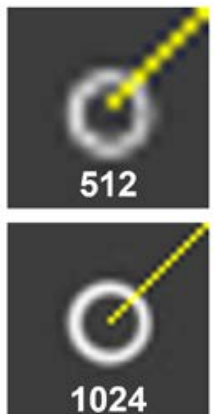

1024

C
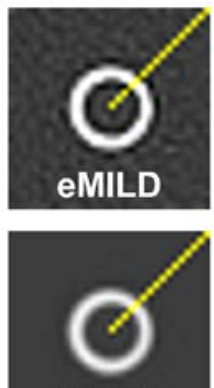

FIRST

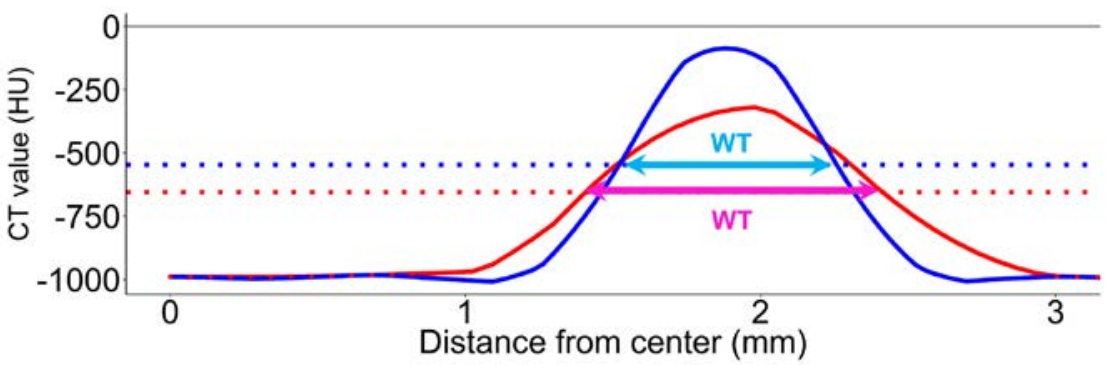

—FIRST512 - FIRST1024

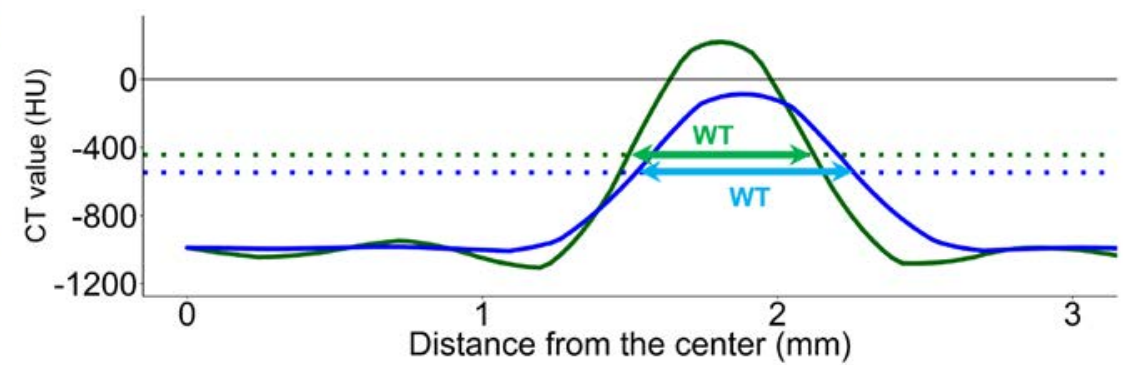

—eMILD1024 —FIRST1024 
Fig. 3
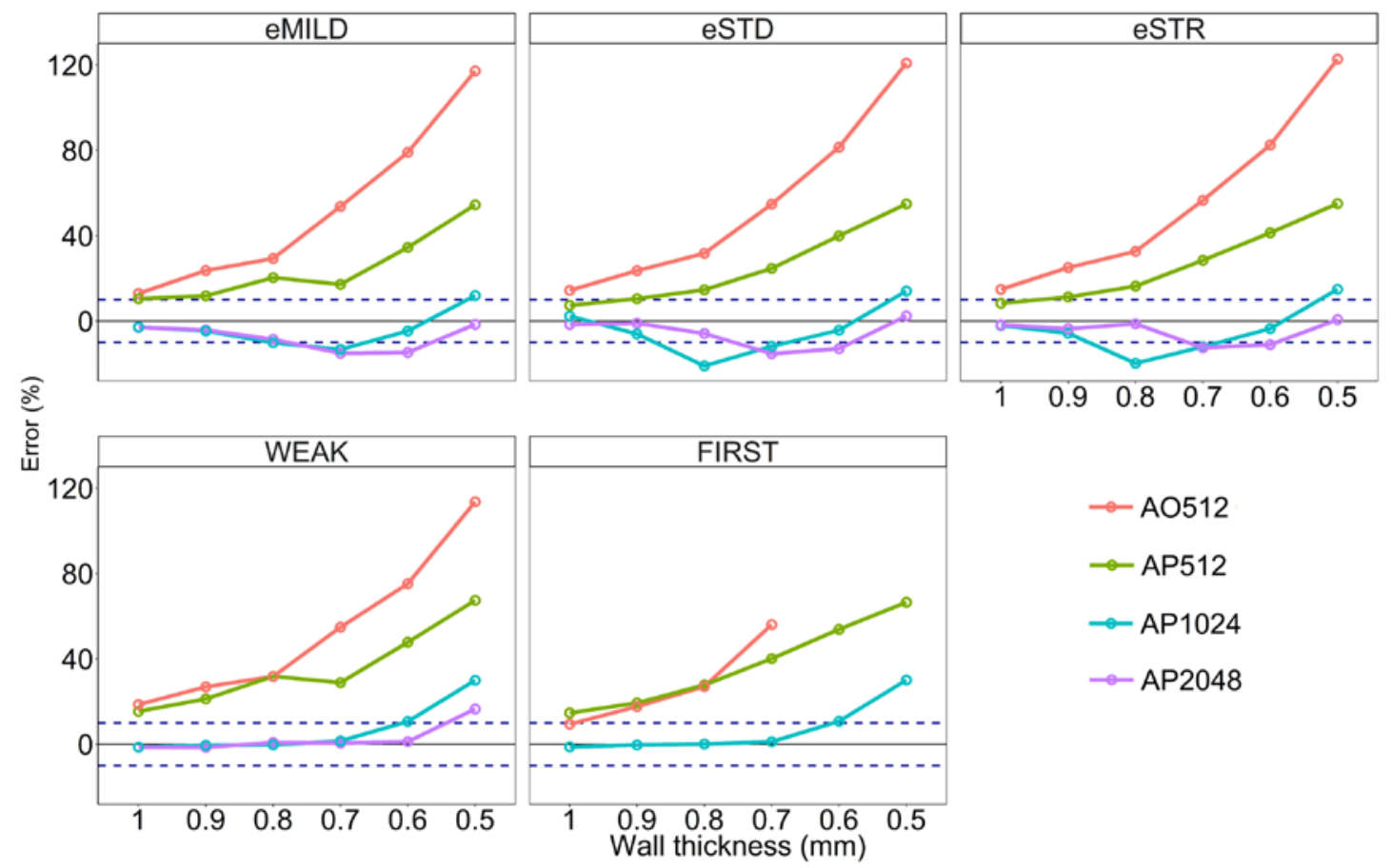

Fig. 4

A

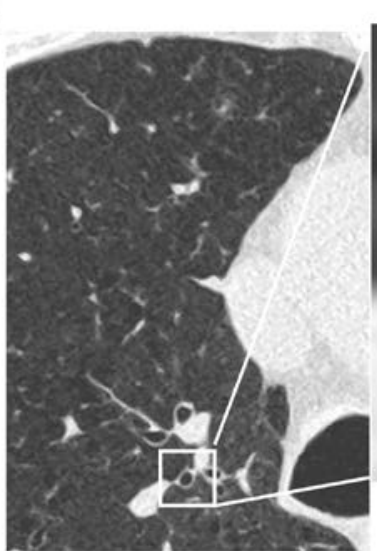

C

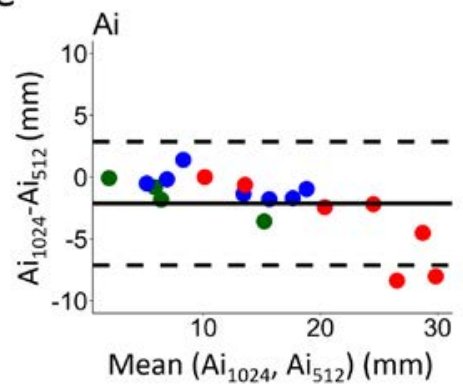

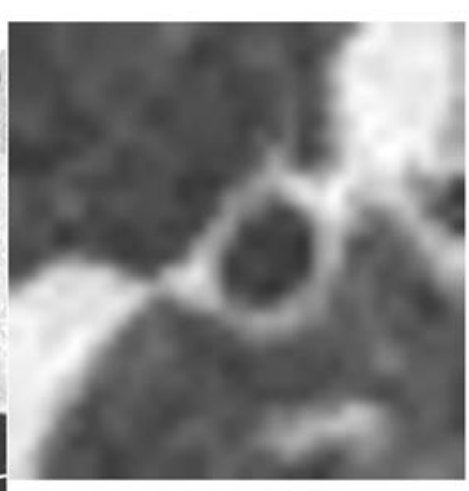

$1024 \times 1024$
B

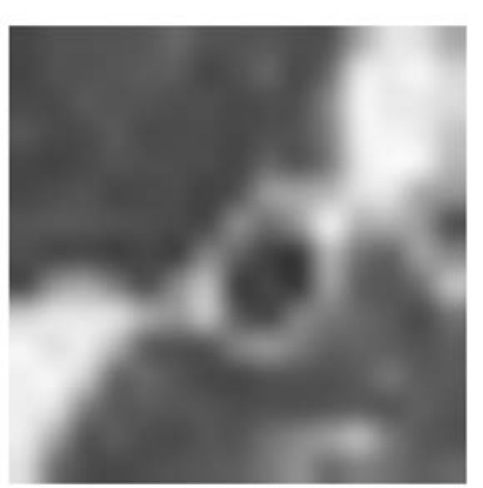

$512 \times 512$

D

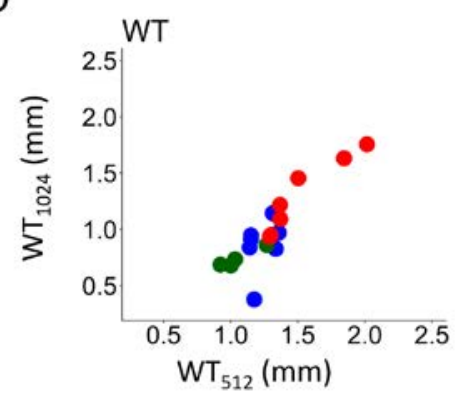

$\bullet$ rdd $\bullet$ th $\bullet 5$ th

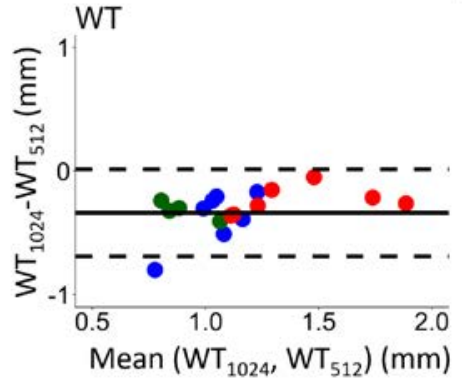

3rd 9 th 5 th 